\title{
From power law intermittence to macroscopic coherent regime
}

\author{
Mauro Bologna, ${ }^{1,2, a)}$ Adrián A. Budini, ${ }^{1,3}$ Filippo Giraldi, ${ }^{1}$ and Paolo Grigolini ${ }^{1,4}$ \\ ${ }^{1}$ Center for Nonlinear Science, University of North Texas, P.O. Box 311427, \\ Denton, Texas 76203-1427, USA \\ ${ }^{2}$ Instituto de Alta Investigación, Universidad de Tarapacá-Casilla, 6-D Arica, Chile \\ ${ }^{3}$ Consejo Nacional de Investigaciones Científicas y Técnicas, Centro Atómico Bariloche, \\ Av. E. Bustillo Km 9.5, 8400 Bariloche, Argentina \\ ${ }^{4}$ Dipartimento di Fisica “E. Fermi," Universitá di Pisa, Largo Pontecorvo, 3, 56127 Pisa, Italy and \\ Istituto dei Processi Chimico Fisici del CNR Area della Ricerca di Pisa, Via G. Moruzzi 1, 56124 Pisa, Italy
}

(Received 21 April 2009; accepted 1 June 2009; published online 25 June 2009)

\begin{abstract}
We address the problem of establishing which is the proper form of quantum master equation generating a survival probability identical to that corresponding to the nonergodic sequence of "light on" and "light off" fluorescence fluctuations in blinking quantum dots. We adopt a theoretical perspective based on the assumption that the abrupt transitions from the light on to light off state are the results of many collisions between system and environment, properly described by the Lindblad equation, and that between two consecutive collisions the system dynamics are frozen. This generates a quantum master equation belonging to the recently proposed class of generalized Lindblad equations, with a time convoluted structure, involving in the specific case of this paper both the unitary and the nonunitary contribution of the Lindblad equation. This is the property that under the low-frequency condition makes the new class of generalized Lindblad equation generates the required survival probability. We make the conjecture that this equation corresponds to the cooperative dynamics of many units that, in isolation, are described by the ordinary Lindblad equation. When the time scale of the unitary term of the Lindblad equation is shorter than the dephasing time, the cooperation generates a surprisingly extended macroscopic coherence.
\end{abstract}

(C) 2009 American Institute of Physics. [DOI: 10.1063/1.3156807]

\section{INTRODUCTION}

The motivation for the research work illustrated in this paper is multiple, but in a way or another all the reasons that led us to this study are related to the increasing interest for complexity. ${ }^{1}$ We believe that the most attractive definition of complexity is the study of the emergent properties of interacting units that cannot be predicted in terms of the properties of the single components due to the nonlinear nature of the interaction between components (see, for instance, the work of Cotsaftis ${ }^{2}$ ). The interacting single units obey an ordinary condition of statistical physics, for instance, the Poisson statistics of the units of Ref. 3 , but the emergent properties manifest a deviation from this ordinary statistics. In the example of Ref. 3 a single unit, in isolation, remains in one of two possible states for a time selected from an exponential distribution density. It is therefore a sort of elementary dipole flipping up and down, with an exponential waiting time distribution density $\psi(\tau)$. However, when the cooperation parameter is switched on, the transition probability from the state $|1\rangle(|-1\rangle)$ to the state $|-1\rangle(|1\rangle)$ is determined also by the other units. It is favored if the majority of the other units are already in the state $|-1\rangle(|1\rangle)$. Otherwise it is reduced. As a result of cooperation, the whole system behaves as a single macroscopic dipole, whose waiting time distribution density $\psi(\tau)$ is proportional to $1 / \tau^{\mu}$ with $\mu<2(\mu=1.5$ in the case of Ref. 3). Thus, assigning the symbol 1 to the dipole up and

${ }^{a)}$ Electronic mail: mauro.bologna@unt.edu. the symbol -1 to the dipole down, we obtain a symbolic sequence whose Kolmogorov-Sinai entropy requires a special treatment. ${ }^{4}$ If we identify the state $|1\rangle$ with the fluorescence "on" and the state $|-1\rangle$ with the fluorescence "off," we can apply this theoretical perspective to the dynamics of nanoscale biological ${ }^{5,6}$ and physical ${ }^{3}$ systems.

The authors of Ref. 5 noted that the sudden jumps of the luminescence signals of synchronized set of molecules reflect the discrete nature of quantum mechanical measurement, and this observation leads us to address this issue using a quantum mechanical formalism. This observation not only generates the crucial issue of adopting a quantum mechanical treatment but also the very delicate one of supplementing this quantum mechanical theoretical approach with a prescription simulating the wave-function collapse. ${ }^{7}$

In other words, the conjecture that the inverse power law nature of the waiting time distribution density is the consequence of cooperation among many units is not enough to afford a quite satisfactory picture of these processes: We have also to explain the emergence of a symbolic sequence revealed by the experiments, with the same entropic properties as those discussed in the very recent paper by Korabel and Barkai. ${ }^{4}$

Which is the quantum master equation equivalent to a nonergodic symbolic sequence? This is a tough problem because the derivation itself of a quantum master equation is still an unsettled problem, in spite of remarkable work done in the last 20 years, since the publication of the celebrated 
paper by Leggett et al. ${ }^{8}$ One of the main problems concerns positivity of the density matrix $\rho(t)$. Even the popular Redfield equation, in spite of being Markov, violates positivity, as pointed out by Suárez et al. ${ }^{9}$ The master equations known to be secure are of the completely positive dynamical semigroup form, ${ }^{10}$

$$
\frac{d}{d t} \rho(t)=-\frac{l}{\hbar}[\hat{H}, \rho(t)]-L_{D} \rho(t),
$$

where $L_{D}$ is the Lindblad superoperator defined by ${ }^{11}$

$$
L_{D} \rho(t)=\frac{1}{\tau_{D}}[\hat{q},[\hat{q}, \rho(t)]],
$$

with $\hat{q}$ being the system's variable measured by the environment and $\tau_{D}$, a symbol used throughout this paper, is the time scale of the environment-induced measurement. The Hamiltonian $\hat{H}$ and the observable $\hat{q}$ are properties of the system of interest, and the hat indicates that they are operators, as prescribed by quantum mechanics. From a mathematical point of view $L_{D}$ is a superoperator, namely, an operator acting on another operator, the density matrix $\rho$. For notational simplicity we do not assign to either of them a special notation to point out their superoperator and operator nature, respectively.

As we shall see in this article, the adoption of Eq. (1) generates a time evolution that is statistically equivalent to the production of a Poisson symbolic sequence that agrees very well with the theoretical arguments used by Cook and Kimble $^{12}$ to account for the quantum jumps revealed by the experiments of Dehmelt and co-workers. ${ }^{13-15}$ It is remarkable that this theoretical scheme, adequate to explain the quantum jumps of single atoms, may be used also to explain the macroscopic quantum jumps recently revealed by $\mathrm{Yu}$ et $a l .{ }^{16}$ Yet, the experimental results in quantum dots, nanorods, and nanowires, recently reviewed and theoretically discussed by Frantsuzov et al. ${ }^{17}$ generate symbolic sequences with the same inverse power law behavior as that leading to the vanishing Lyapunov coefficients of Ref. 4.

How to account for this intriguing fact? One may make the conjecture that the problem is settled by turning Eq. (1) into a time convoluted equation suggesting the existence of memory. Let us disregard, for the time being, the unitary contribution to Eq. (1). To a first sight, an attractive way to add memory to the memoryless Lindblad structure rests on the time convoluted structure,

$$
\frac{d}{d t} \rho(t)=-\tau_{D} \int_{0}^{t} \Phi\left(t-t^{\prime}\right) L_{D}\left(t^{\prime}\right) \rho\left(t^{\prime}\right) d t^{\prime},
$$

with $\Phi(t)$ being a memory kernel, taking into account that the environment of the system of interest is not infinitely fast. Barnett and Stenholm ${ }^{18}$ showed, however, that even the choice of an exponentially decaying memory generates nonphysical behavior. Only when the exponential decay is very fast, this picture produces sensible and reliable results. In this case, the resulting operator is essentially equivalent to the Lindblad operator. The conclusions of Barnett and Stenholm seem to be in line with a proposal made by Wilkie ${ }^{19}$ to ensure the positivity condition. Wilkie, who took the unitary contribution into account, adopted the following nonMarkov, or generalized master equation,

$$
\frac{d}{d t} \rho(t)=f(t)-\frac{l}{\hbar}[\hat{H}, \rho(t)]-\tau_{D} \int_{0}^{t} d t^{\prime} \Phi\left(t-t^{\prime}\right) L_{D} \rho\left(t^{\prime}\right) .
$$

To ensure the positivity condition, a crucial role is played by the term $f(t)$, reminiscent of the inhomogeneous term produced by the Zwanzig projection method. ${ }^{20}$ This suggests that the inhomogeneous term predicted by the Zwanzig projection formalism cannot be neglected, this inhomogeneous term being, as well as the non-Markov kernel of Eq. (3), an important memory manifestation.

More recently, one of $\mathrm{us}^{21}$ proposed the structure of Eq. (3), and more specifically, in the presence of a unitary contribution, ${ }^{22}$

$$
L_{U} \rho(t) \equiv \frac{1}{i \hbar}[\hat{H}, \rho(t)]
$$

the form

$$
\frac{d}{d t} \rho(t)=L_{U} \rho(t)-\tau_{D} \int_{0}^{t} d t^{\prime} \Phi\left(t-t^{\prime}\right) L_{D} \exp \left[L_{U}\left(t-t^{\prime}\right)\right] \rho\left(t^{\prime}\right) .
$$

Adopting, however, a theoretical perspective whose theoretical roots, rather than on the Zwanzig projection method, or, more in general, on a prime principle approach, is based on the pioneering work of Montroll and Weiss. ${ }^{23}$ This theory is denoted as continuous time random walk (CTRW). It is possible to derive anomalous diffusion from the ordinary random walk prescription by releasing the assumption that the time distribution between two consecutive jumps events has a Poisson form. The resulting diffusion process is derived from a process of subordination to ordinary random walk. The two successive events considered in Ref. 21 are quantum random collisions of the system with its environment. Reference 21 explored the attractive possibility of creating a non-Markov quantum process by assigning a non-Poisson distribution to the time distance between two successive events produced by the Lindblad prescription. This procedure can be interpreted as a form of subordination to the Lindblad equation. In this paper we use the same perspective of subordination to the Lindblad dynamics with the new vision in mind that the collection of a large number of interacting dipoles may be replaced by a single virtual dipole, whose dynamics are frozen for extended time intervals with the waiting time distribution density $\psi(\tau)$. The earlier mentioned waiting time distribution density $\psi(\tau)$ departs from the exponential form as a consequence of the cooperation among many dipoles, and the set of Cook and Kimble ${ }^{12}$ units is replaced by a single global unit, whose dynamics is driven by the non-Poisson waiting time distribution density $\psi(\tau)$.

The adoption of an approach based on the subordination to Lindblad equation sheds light on why Eq. (3) may fail also with a simple exponential kernel. In fact, as well known, and as we shall see again in Sec. II C, the adoption of the subordination theory yields for the Laplace transform of $\Phi(t)$ 
[note that we shall use throughout the notation $\tilde{f}(u)$ $\equiv \int_{0}^{\infty} \exp (-u t) f(t) d t$ for the Laplace transform of $\left.f(t)\right]$,

$$
\widetilde{\Phi}(u)=\frac{u \widetilde{\psi}(u)}{(1-\widetilde{\psi}(u))} .
$$

The exponential memory kernel of Ref. 18, $\Phi(t)$ $=c^{2} \exp [-\gamma t]$, would generate consequently a function $\psi(t)$ defined by

$$
\frac{u \widetilde{\psi}(u)}{1-\widetilde{\psi}(u)}=\frac{c^{2}}{u+\gamma} .
$$

Solving with respect to $\widetilde{\psi}(u)$ and inverting the Laplace transform, we find ${ }^{21}$

$$
\psi(t)=\frac{2 \exp \left[-\frac{t \gamma}{2}\right] \sinh \left[\frac{1}{2} c t \sqrt{\lambda^{2}-4}\right]}{\sqrt{\lambda^{2}-4}},
$$

where we set $\lambda=\gamma / c$. The function $\psi(t)$ is defined positive if $\lambda \geq 2$. It has to be pointed out that $\Phi(t)$ is not a correlation function and that the relevant physical property is the waiting time distribution density $\psi(t)$. It is not surprising that the violation of the positivity condition of $\psi(t)$ may generate the breakdown of the positivity condition of the density matrix $\rho(t)$. On the contrary, if $\psi(t)$ is definite positive, as a waiting time distribution density ought to be, the density matrix $\rho(t)$ is certainly definite positive, as we shall see in this paper.

In this paper we propose

$$
\frac{d}{d t} \rho(t)=-\theta \int_{0}^{t} d t^{\prime} \Phi\left(t-t^{\prime}\right)\left\{\frac{l}{\hbar}\left[\hat{H}, \rho\left(t^{\prime}\right)\right]+L_{D} \rho\left(t^{\prime}\right)\right\},
$$

where $\theta$ is the time duration of the collision between system and environment. This time duration is extremely small, and the memory kernel of the generalized master equation that we propose is determined by the much larger time distances between two consecutive collisions. As earlier mentioned, the motivation for this generalized form of Lindblad equation rests on the work of Ref. 3. The quantum mechanical counterpart of the single units of Ref. 3 is the term in between curly brackets. In the natural time scale, namely, when the memory kernel $\Phi(t)$ is a delta of Dirac, this term is equivalent to a symbolic sequence with an exponential waiting time distribution density in either the up $(+)$ or down $(-)$ state. Thus, it is equivalent to the Poisson subordination to a fair coin tossing process. As a consequence when the Laplace transform of $\Phi(t)$ is given by Eq. (7), the resulting process is equivalent to the double-subordination approach of Ref. 24, generating a Mittag-Leffler relaxation. The Mittag-Leffler function is a generalization of the conventional exponential relaxation, with the short-time regime described by a stretched exponential relaxation and a long-time regime given by an inverse power law function. As pointed out in Ref. 24, the slower the first subordination function, the more extended the stretched exponential regime. The time scale of the first subordination function is given by $\omega^{2} \tau_{D}$, where $\omega$ is the frequency of the unitary term. Thus, we can reduce this time scale by decreasing $\tau_{D}$ or making more frequent the quantum measurement. ${ }^{25}$

It is important to point out that the adoption of the structure of Eq. (10) rather than that of Eq. (6) prevents us from recovering a general property of virtually any kinetic equation, yielding the bath-free evolution of the density matrix when the system-bath coupling goes to zero. This restriction, on the other hand, is the price to pay to realize a physical condition equivalent to the symbolic sequence of Korabel and Barkai. ${ }^{4}$

We are mainly interested in the case where $\tau_{D}$ is moderately smaller than $\omega^{-1}$ so as to contain the stretched exponential regime within a restricted short-time regime, thereby essentially generating an inverse power law relaxation. This is so because the Blinking Quantum Dot (BQD) produces waiting distribution densities of "on" and "off" states that are inverse power law functions, ${ }^{17}$ whose renewal nature has been thoroughly assessed by means of the aging experiment of Ref. 26. All this leads us to conclude that Eq. (10) is the proper generalized master equation for the intermittent fluorescence of BQD. However, we shall not limit our study to the condition $\omega \tau_{D}<1$ and we shall explore also the condition $\omega \tau_{D} \gg 1$, whose thermodynamic importance has been pointed out by the recent work of Ref. 25. We shall find the interesting result that the cooperation between units that in isolation would generate damped oscillations extends coherence to a much wider time scale, thereby producing an effect referred to as macroscopic coherence. The emergence of macroscopic coherence from a stochastic procedure based on the random choice of waiting times is, to some extent, surprising, and in the lights of the results of Ref. 25 might have interesting applications.

The outline of the paper is as follows. In Sec. II we derive Eq. (1) using a generalization of the method proposed by the authors of Ref. 27, and by means of heuristic arguments we prove that it is equivalent to a generator of a Poisson symbolic sequence, provided that the time scale of the inphasing term ${ }^{28}$ is much larger than the time scale of the dephasing term. In Sec. III we show that, as expected, in the same physical condition, Eq. (10) generates an inverse power law relaxation. We explore also the physical condition generating macroscopic coherence. Section IV is devoted to a concise review of the earlier work ${ }^{21}$ for the purpose of stressing the importance of replacing the structure of Eq. (6) with the new form of Eq. (10). Finally, in Sec. V we discuss the relevance that this result may have for the physics of blinking quantum dots and for further theoretical and experimental research work in this field.

\section{QUANTUM MASTER EQUATION}

In this section we illustrate a physical derivation of the Lindblad equation, and we show that moving from $\omega \tau_{D}<1$ to $\omega \tau_{D}>1$ corresponds to turning the quantum Zeno effect (QZE) into the anti-Zeno effect (AZE). ${ }^{25}$ This is an already known phenomenon, see Ref. 25 and references therein, but it serves the basic purpose of preparing the ground for our general discussion of the cooperative-induced subordination effect. 


\section{A. Lindblad equation}

Let us derive first of all the Lindblad equation by using the physical approach of Ref. 27 . We call $\hat{H}$ the object's Hamiltonian and we denote by $\hat{q}$ the object's variable that is measured by the environment. We imagine that the object collides at regular times with bath particles, a particle at a time, and that there is no correlation among different collisions. The time duration of a collision is $\theta$ and it is very short. The $n$th collision corresponds to the Hamiltonian $\hat{H}_{n}$ $-\hat{q}_{n} \hat{y}_{n}$, where $\hat{y}_{n}$ is, for instance, the coordinate of the bath particle. The time evolution operator corresponding to this collision is

$$
\hat{U}_{n}=\exp \left[\frac{\hat{H}_{n}-\hat{q}_{n} \hat{y}_{n}}{i \hbar} \theta\right] .
$$

Thanks to the assumption that $\theta$ is very small, we adopt the Taylor series expansion,

$$
\hat{U}_{n}=1+\frac{\theta}{i \hbar}\left(\hat{H}_{n}-\hat{q}_{n} \hat{y}_{n}\right)-\frac{\theta^{2}}{2 \hbar^{2}}\left(\hat{H}_{n}-\hat{q}_{n} \hat{y}_{n}\right)^{2} .
$$

The effect of a collision is to make the transition from $\rho_{n}$ to $\rho_{n+1}$ as described by the following prescription:

$$
\rho_{n+1}=\operatorname{Tr}_{B}\left(\hat{U}_{n}\left|\psi_{n}\right\rangle \rho_{n}\left\langle\psi_{n}\right| \hat{U}_{n}^{+}\right) .
$$

The explanation of Eq. (13) goes as follows. At the very moment of the collision the bath density matrix $\left|\psi_{n}\right\rangle\left\langle\psi_{n}\right|$ and the object's density matrix $\rho_{n}$ are statistically independent. They are correlated by the collision event due to the interaction $-\hat{q}_{n} \hat{y}_{n}$. At the end of the collision process we make a trace over a complete set of bath states so as to define the new density matrix of the system of interest (the object according to Ref. 27). By plugging Eq. (12) into Eq. (11) we obtain

$$
\rho_{n+1}=\rho_{n}+\theta \hat{A}+\theta^{2} \hat{B}
$$

where

$$
\hat{A} \equiv \frac{\left[\hat{H}_{n}-\hat{q}\left\langle\hat{y}_{n}\right\rangle, \rho_{n}\right]}{i \hbar}
$$

and

$$
\hat{B} \equiv-\frac{\left\{\left[\hat{H}_{n},\left[\hat{H}_{n}, \rho_{n}\right]\right]-\left\langle\hat{y}_{n}\right\rangle\left[\hat{H}_{n},\left[\hat{q}_{n}, \rho_{n}\right]\right]\right\}+\left\{\left[\hat{q}_{n},\left[\hat{H}_{n}, \rho_{n}\right]\right]+\left\langle\hat{y}_{n}^{2}\right\rangle\left[\hat{q}_{n},\left[\hat{q}_{n}, \rho_{n}\right]\right]\right\}}{2 \hbar^{2}} .
$$

We now make the approximation $\left\langle\hat{y}_{n}\right\rangle=0$ (no bias). Furthermore, we make the strong-collision approximation that although $\theta$ is vanishing small, the quantity

$$
\sigma_{n}^{2}=\theta\left\langle\hat{y}^{2}\right\rangle
$$

is finite. Thus, we obtain

$$
\rho_{n+1}=\left(1+\theta K_{n}\right) \rho_{n},
$$

where

$$
K_{n} \rho_{n}=\frac{1}{i \hbar}\left[\hat{H}_{n}, \rho_{n}\right]-\frac{\sigma_{n}^{2}}{2 \hbar^{2}}\left[\hat{q}_{n},\left[\hat{q}_{n}, \rho_{n}\right]\right] .
$$

We assume

$$
\theta \ll \tau_{D} \equiv \frac{2 \hbar^{2}}{\sigma^{2}} .
$$

Thus for times $t \gg \theta$, but not necessarily larger than $\tau_{D}$, we may adopt the continuum time representation, and, assuming $\sigma_{n}$ and $H$ to be independent of time, we obtain

$$
\frac{\partial}{\partial t} \rho(t)=K \rho(t),
$$

where

$$
K=L_{U}-L_{D} .
$$

In the case here under study, the generator of unitary time evolution and the environment-induced measurement generator $L_{D}$ have the form [for the sake of clarity we rewrite here Eqs. (5) and (2)],

$$
\begin{aligned}
& L_{U} \rho(t) \equiv \frac{1}{i \hbar}[\hat{H}, \rho(t)], \\
& L_{D} \rho(t)=\frac{1}{\tau_{D}}[\hat{q},[\hat{q}, \rho(t)]],
\end{aligned}
$$

respectively. Equation (21) with the superoperator $K$ defined by Eqs. (22)-(24) is the celebrated Lindblad equation. ${ }^{11}$

Note that the inequality of Eq. (20) makes it possible for us to consider the time region between $\theta$ and $\tau_{D}$ as well as the time region $t>\tau_{D}$.

\section{B. On the equivalent Poisson sequence}

For a heuristic discussion of this important aspect, let us consider the Hamiltonian,

$$
\hat{H}_{T}=\hat{H}-\hat{q} \hat{y},
$$

where 


$$
\hat{H} \equiv(|1\rangle\langle-1|+|-1\rangle\langle 1|) V
$$

and

$$
\hat{q} \equiv|1\rangle\langle 1|-|-1\rangle\langle-1| .
$$

In the continuous time representation with $t \gg \theta$, the coherent contribution to the time evolution of the density matrix $\rho$ corresponds to the wave-function time evolution driven by the Hamiltonian $\hat{H}$,

$$
|\psi(n)\rangle=\cos (\omega t)|1\rangle-i \sin (\omega t)|-1\rangle, \quad \omega \equiv \frac{V}{\hbar} .
$$

Let us imagine that the system moves from the initial condition $|\psi(0)\rangle=|1\rangle$. Let us assume that the dephasing process is equivalent to the wave-function collapse ${ }^{7}$ and that the environment-induced measurement occurs at times $\tau_{D}$, $2 \tau_{D}, \ldots, n \tau_{D}, \ldots$, with $\tau_{D}$ so short as to ensure the condition $\omega \tau_{D} \ll 1$. In this case, $\left|\cos \left(\omega \tau_{D}\right)\right|^{2} \approx 1$ and $\left|\sin \left(\omega \tau_{D}\right)\right|^{2}$ $\approx\left(\omega \tau_{D}\right)^{2} \ll 1$. Let us consider the time scale $t=n \tau_{D} \gg \tau_{D}$, i.e., $n \gg 1$. Thus, the survival probability $\Psi(t)$, namely, the probability that the system is still in the state $|1\rangle$ up to time $t=n \tau_{D}$ is

$$
(1-g)^{n}=\exp [n \log (1-g)] \approx \exp (-g n),
$$

where

$$
g \equiv\left(\omega \tau_{D}\right)^{2} .
$$

In conclusion, we have

$$
\Psi(t)=\exp \left(-\omega^{2} \tau_{D} t\right) .
$$

We see that the more frequent the measurement process, namely, the smaller the dephasing time $\tau_{D}$, the more extended the sojourn time in the state $|1\rangle$. This phenomenon is called QZE. $^{25,29}$

It is important to notice that the solution of Eq. (21) yields the same result. In fact, rather than using $\rho_{i, j} \equiv\langle i|\rho| j\rangle$, let us use the variables

$$
\Pi(t) \equiv \rho_{1,1}-\rho_{-1,-1}
$$

and

$$
R(t) \equiv \rho_{1,-1}-\rho_{-1,1} .
$$

Then, Eq. (21) yields

$$
\frac{d \Pi}{d t}=2 i \omega R
$$

and

$$
\frac{d R}{d t}=2 i \omega \Pi-\frac{4}{\tau_{D}} R,
$$

which, thanks to Eq. (34), reads

$$
\frac{d R}{d t}=2 i \omega \Pi+i \frac{2}{\omega \tau_{D}} \frac{d \Pi}{d t} .
$$

By differentiating Eq. (34) with respect to time and plugging into it Eq. (36), we obtain

$$
\frac{d^{2} \Pi}{d t^{2}}+4 \omega^{2} \Pi+\frac{4}{\tau_{D}} \frac{d}{d t} \Pi=0 .
$$

This is the well known equation for a damped harmonic oscillator, yielding in the QZE regime $\left(\omega \tau_{D}<2\right)$ an exponential relaxation regime that for $\omega \tau_{D} \ll 1$ becomes

$$
\frac{d \Pi}{d t}=-\omega^{2} \tau_{D} \Pi,
$$

whose solution is

$$
\frac{\Pi(t)}{\Pi(0)}=\exp \left(-\omega^{2} \tau_{D} t\right)
$$

which coincides with Eq. (31).

The condition $\omega \tau_{D}=1$ is the boundary between the QZE and the AZE. ${ }^{25}$ In the work of Ref. 30 it was established that the condition $\tau_{D}<1 / \omega$ makes the Shannon entropy increase with the number of collision-induced wave-function collapses. According to the authors of Ref. 25 the condition $\omega_{T} \tau_{D}>1$ corresponds to the AZE regime, and consequently to an antientropic behavior.

\section{From the natural time $\boldsymbol{n}$ to the continuous time $\boldsymbol{t}$}

Let us now make the assumption that the single Cook and Kimble units undergo a cooperative interaction as well as an interaction with the measuring bath that we can imagine similar to the bath of harmonic oscillators of Ref. 25. In this case, adopting the suggestion emerging from the results of Ref. 3, we conjecture that a global unit is created with the same dynamics as the single units, but the time interval between two consecutive collisions is now drawn from the distribution density,

$$
\psi(\tau)=(\mu-1) \frac{T^{\mu-1}}{(\tau+T)^{\mu}} .
$$

In the case of Ref. 3, $\mu=1.5$. However, for the sake of generality here we make a general discussion, and later we shall refine the calculations by discussing the specific case of Eq. (40), with $\mu<2$.

At time $t$, the density matrix $\rho(t)$ becomes

$$
\rho(t)=\sum_{n=0}^{\infty} \int_{0}^{t} d t^{\prime} \psi_{n}\left(t^{\prime}\right) \Psi\left(t-t^{\prime}\right)(1+\theta K)^{n} \rho(0) .
$$

Note that $\psi_{n}(t)$ is the probability that $n$ events occurred, the last of which at time $t$. The function $\Psi(t)$ denotes the probability that no event occurs up to time $t$ and is given by

$$
\Psi(t)=\int_{t}^{\infty} d t^{\prime} \psi\left(t^{\prime}\right) .
$$

The Laplace transforms of both functions $\psi_{n}(t)$ and $\Psi(t)$ can be expressed in terms of the Laplace transform of $\psi(t)$. The time evolution of $\rho(t)$ depends on the events. The occurrence of an event corresponds to activating the superoperator 1 $+\theta K$. The occurrence of $n$ events turns the initial condition $\rho(0)$ into $(1+\theta K)^{n} \rho(0)$. This form is kept from time $t^{\prime}$ at which the last collision occurs, up to time $t$, the time interval from $t^{\prime}$ to $t$ being characterized by no event occurring. Of 
course, the expression of Eq. (41) takes into account that the number of possible events may range from the no event to the infinitely many event condition. For this mathematical idealization to be as realistic as possible, we have to assume that the waiting times $\tau$ may be arbitrarily small, as small as we wish. This is not quite realistic, insofar as there may be a bottom truncation time. In real systems there exists also a top truncation time. We assume the latter to be so big as to make it possible to accommodate a very large (virtually infinite) number of collisions.

Adopting the procedure of the users of CTRW (Ref. 23) (see, for example, Refs. 31-34), we prove that Eq. (41) generates the time convoluted master equation of Eq. (10), which is the generalization of Lindblad equation proposed in this article. Adopting the language of subordination theory, ${ }^{31-34}$ we refer to Eq. (41) as a transformation from the natural time, where the Lindblad equation applies, to the physical time, corresponding to the experimental observation.

It is important to notice that $\rho(t)$ generated by Eq. (41) is certainly definite positive, being the sum of infinite terms, each of which is a definite positive density matrix. As a consequence, Eq. (10), being equivalent to Eq. (41), is a generalized form of Lindblad equation fulfilling the condition of producing a definite positive density matrix. Of course, the same arguments have been used ${ }^{21}$ to prove that Eq. (6) fits this important requirement. We are reinstating all this for the sake of clarity.

\section{FROM POWER LAW INTERMITTENCE TO MACROSCOPIC COHERENT REGIME}

Let us consider the total Hamiltonian $\hat{H}_{T}=\hat{H}-\hat{q} \hat{y}$, where

$$
\hat{H} \equiv(|1\rangle\langle-1|+|-1\rangle\langle 1|) V, \quad \hat{q} \equiv(|1\rangle\langle 1|-|-1\rangle\langle-1|) .
$$

In this case the density matrix of interest is two dimensional. Equivalently, we can consider its elements as a fourdimensional vector and its evolution in the discrete time representation is

$$
\mathbf{r}(n+1)=(1+\theta \mathbf{M}) \mathbf{r}(n),
$$

with

$$
\mathbf{r}(n) \equiv\left[\begin{array}{c}
\rho_{1,1} \\
\rho_{1,-1} \\
\rho_{-1,1} \\
\rho_{-1,-1}
\end{array}\right], \quad \mathbf{M}=\left[\begin{array}{cccc}
0 & \imath \omega & -\imath \omega & 0 \\
\imath \omega & -\frac{4}{\tau_{D}} & 0 & -\imath \omega \\
-\imath \omega & 0 & -\frac{4}{\tau_{D}} & \imath \omega \\
0 & -\imath \omega & \iota \omega & 0
\end{array}\right],
$$

and we set $\omega=V / \hbar, \tau_{D}^{-1}=\sigma^{2} / 2 \hbar^{2}$. The eigenvalues of $\mathbf{M}$ are

$$
\begin{aligned}
& \lambda_{1}=0, \quad \lambda_{2}=-\frac{4}{\tau_{D}}, \quad \lambda_{3}=-\frac{2}{\tau_{D}}\left[1+\sqrt{1-\tau_{D}^{2} \omega^{2}}\right], \\
& \lambda_{4}=\frac{2}{\tau_{D}}\left[-1+\sqrt{1-\tau_{D}^{2} \omega^{2}}\right] .
\end{aligned}
$$

To double check this formal procedure, let us prove that Eq. (44) yields a result equivalent to Eq. (31). In fact, the solution $\mathbf{r}(n)$ is

$$
\mathbf{r}(n)=(1+\theta \mathbf{M})^{n} \mathbf{r}(0) .
$$

Writing $\mathbf{r}(0)$ as $\mathbf{r}(0)=\sum_{i=1}^{4} c_{i} \mathbf{v}_{i}$, where $\mathbf{v}_{i}$ are the eigenvectors of the matrix $\mathbf{M}$, we have

$$
\mathbf{r}(n)=\sum_{i=1}^{4} c_{i}\left(1+\theta \lambda_{i}\right)^{n} \mathbf{v}_{i} \approx \sum_{i=1}^{4} c_{i} \exp \left[n \theta \lambda_{i}\right] \mathbf{v}_{i} .
$$

Choosing as initial condition $\mathbf{r}(0)=[1,0,0,0]$, in the limit $\omega \tau_{D} \ll 1$ and $n \rightarrow \infty$, we have that

$$
\Pi(t)=\rho_{1,1}(t)-\rho_{-1,-1}(t)=\exp \left[-\tau_{D} \omega^{2} n \theta\right] .
$$

Setting $t=n \theta$, we get Eq. (31). Note that the heuristic arguments behind Eq. (31) correspond to the adoption of the coarse-grained time $t=n_{D} \tau_{D}$, whereas here we use the time $t=n_{\theta} \theta$. The condition

$$
n_{\theta} \theta=n_{D} \tau_{D}
$$

and the inequality of Eq. (20) indicate that to realize $n_{D}$ wave-function collapses, a much larger number of Lindblad collisions, $n_{\theta}=n_{D}\left(\tau_{D} / \tau_{\theta}\right) \gg n_{D}$, is required.

Using this setting and performing the subordination, we obtain

$$
\widetilde{\mathbf{r}}(u)=\frac{1}{u} \frac{1-\tilde{\psi}(u)}{1-\left(1+\tau_{D} \mathbf{M}\right) \tilde{\psi}(u)} \mathbf{r}(0),
$$

where $\tilde{\psi}(u)$, according to the notation adopted throughout, is the waiting time distribution in the Laplace representation. We assume for $\tilde{\psi}(u)$ the Laplace transform of the negative derivative of a Mittag-Leffler function, namely,

$$
\widetilde{\psi}(u)=\frac{1}{1+T^{\mu-1} u^{\mu-1}},
$$

with $\mu<2$ and $T$ is a time parameter as in Eq. (40). Asymptotically we have that $\psi(t) \approx-\left(T^{\mu-1} / \Gamma[1-\mu]\right) t^{-\mu}$. After straightforward algebra we obtain

$$
\tilde{\mathbf{r}}(u)=\sum_{i=1}^{4} \frac{u^{\mu-2}}{u^{\mu-1}-\frac{\tau_{D}}{T^{\mu-1}} \lambda_{i}} c_{i} \mathbf{v}_{i} .
$$

Equation (53) can be inverted, and the final result is

$$
\mathbf{r}(t)=\sum_{i=1}^{4} c_{i} E_{\mu-1}\left[\tau_{D} \lambda_{i}\left(\frac{t}{T}\right)^{\mu-1}\right] \mathbf{v}_{i},
$$

where $E_{\mu-1}[z]$ is the Mittag-Leffler function. Choosing $\mathbf{r}(0)=[1,0,0,0]$ then we obtain 


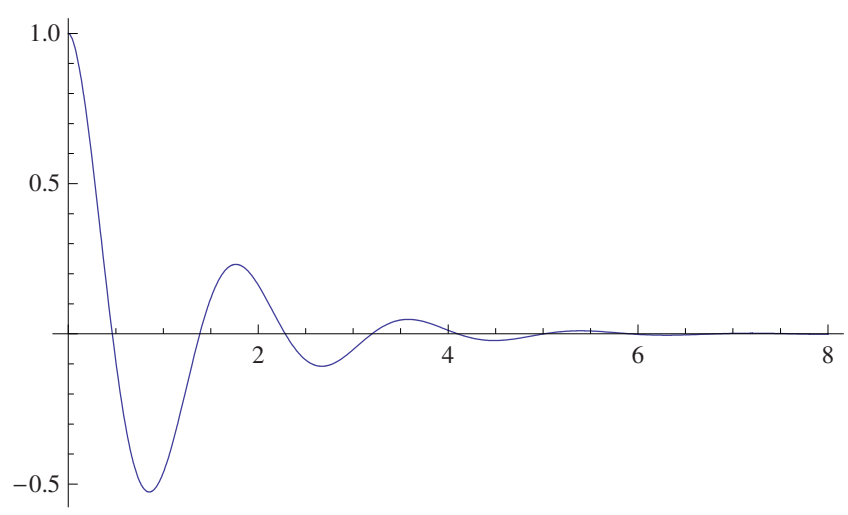

FIG. 1. (Color online) The plot of Eq. (55) for $\omega>\omega_{\mathrm{cr}}$. The numerical values of the parameters are $\tau_{D}=1, \omega=5 \pi, \mu=1.9$, and $a=10$.

$$
\begin{aligned}
\Pi(t)= & \rho_{1,1}(t)-\rho_{-1,-1}(t) \\
= & {\left[\frac { 1 } { 2 } ( 1 + \frac { 1 } { \sqrt { 1 - \tau _ { D } ^ { 2 } \omega ^ { 2 } } } ) E _ { \mu - 1 } \left[2\left(-1+\sqrt{1-\tau_{D}^{2} \omega^{2}}\right)\right.\right.} \\
& \left.\times\left(\frac{t}{T}\right)^{\mu-1}\right]+\frac{1}{2}\left(1-\frac{1}{\sqrt{1-\tau_{D}^{2} \omega^{2}}}\right) E_{\mu-1} \\
& \left.\times\left[-2\left(1+\sqrt{1-\tau_{D}^{2} \omega^{2}}\right)\left(\frac{t}{T}\right)^{\mu-1}\right]\right] .
\end{aligned}
$$

For $\omega \tau_{D} \ll 1$ and $t \rightarrow \infty$ we obtain

$$
\Pi(t) \approx E_{\mu-1}\left[-\tau_{D}^{2} \omega^{2}\left(\frac{t}{T}\right)^{\mu-1}\right] .
$$

From Eq. (55) we can also note that there exists a critical value of the frequency $\omega_{\mathrm{cr}}=\tau_{D}^{-1}$. For $\omega>\omega_{\mathrm{cr}}$ the arguments of the Mittag-Leffler function are complex so that it can yield exponentially damped oscillatory behavior as well as an inverse power law contribution, ${ }^{35,36}$ as shown in Fig. 1. An analytical expression in terms of exponential functions is found for $\Pi(t)$ in the asymptotic limit $t \rightarrow \infty$ and $\omega>\omega_{\mathrm{cr}}$, namely,

$$
\begin{aligned}
\Pi(t) \approx & \left.\frac{\exp \left[t|z|^{1 / \mu-1} \cos \frac{\phi}{\mu-1}\right]}{\mu-1}\right] \cos \left(t|z|^{1 / \mu-1} \sin \frac{\phi}{\mu-1}\right) \\
& \left.+\frac{\sin \left(t|z|^{1 / \mu-1} \sin \frac{\phi}{\mu-1}\right)}{\sqrt{\tau^{2} \omega^{2}-1}}\right]+\frac{\frac{\sin \phi}{\sqrt{\tau^{2} \omega^{2}-1}}-\cos \phi}{|z| \Gamma[2-\mu] t^{\mu-1}},
\end{aligned}
$$

where, for compactness, we introduced the notation

$$
z \equiv|z| \exp [\imath \phi]=\frac{2\left(-1+\imath \sqrt{\tau_{D}^{2} \omega^{2}-1}\right)}{T^{\mu-1}} .
$$

Let us stress two important issues.

There are two regimes for the survival probability. For $\omega<\omega_{\mathrm{cr}}$, corresponding to the QZE regime, the survival probability is asymptotically a power law, namely, $\Pi(t) \sim t^{1-\mu}$ [see Eq. (56)]. For $\omega>\omega_{\mathrm{cr}}$, corresponding to the AZE regime, the survival probability is a mixture of a seemingly Poissonian contribution (the exponentially damped oscillations) and an inverse power law relaxation [see Eq. (57)]. The Poissonian-like part is dominant with respect to the power law in a time range that could be very extended. If we imagine a hypothetic experiment, this time window could be extended as much as the duration of the experiment itself.

(ii) The frequency of Poissonian-like contribution to $\Pi(t)$ is different from the frequency $\omega$ of the unitary term of the Hamiltonian. Indeed the Poisson-like part has a frequency $\Omega=|z|^{1 /(\mu-1)} \sin (\phi / \mu-1)$ and a damping $\Gamma=|z|^{1 /(\mu-1)} \cos (\phi / \mu-1)$. The cooperation between units is expected to make the parameter $T$ of Eq. (52) very large and consequently the frequency $\Omega$ much smaller than the frequency $\omega$.

It is important to stress that to establish under which conditions a sequence of Lindblad collisions is compatible with the production of symbolic sequences, with either a finite or a vanishing Lyapunov coefficient, we have to use Eq. (50). Setting this condition is equivalent to assuming that $t=\tau_{D}$ is the minimum time step, thereby making it impossible for this statistical equivalence to apply also to the time scale $t<\tau_{D}$. In other words, the emergence of damped oscillatory behavior of Eq. (57) is incompatible with the existence of an equivalent symbolic sequence. On the other hand, we cannot rule out the existence of the attractive condition discussed in Ref. 25. In this case, the condition $\tau_{D}>1 / \omega$ is physically accessible, but we have to rule out the possibility of creating a symbolic sequence, corresponding in the natural time scale to the Shannon entropy linearly increasing with the number of measurement-induced wave-function collapses. In this case the results of this paper may be interpreted as the indication that the AZE, generating anti-rather than entropic ${ }^{30}$ effect, may be amplified by cooperation so as to produce macroscopic effects.

\section{ON THE LINEAR SUPERPOSITION OF ORDINARY UNITARY TIME EVOLUTION AND SPORADIC MEASUREMENT}

The results of Ref. 18 raised some interest to find nonMarkovian master equations that lead to completely positive evolutions and the microscopic interactions from where they arise as well. In Ref. 37, it was demonstrated that strong non-Markovian effects may arise from a system interacting with a reservoir whose dynamic action can be represented by a direct sum of Markovian subreservoirs as well as from systems whose interaction with the bath is mediated by extra degrees of freedom.

The time evolutions associated with these dynamics ${ }^{37}$ fulfill the positivity condition. Under a noncontrolled effective approximation, ${ }^{22}$ we derive these time evolutions from a generalized master equation with a structure similar but not equivalent to that of Eq. (10). This is, in fact, the reason that motivated the search of this paper for an alternative stochastic formulation. In Ref. 37 the dynamics of the system between the crucial events are the unitary process $\exp \left[t L_{U}\right]$, where the Liouvillan $L_{U}$ follows from Eq. (23). The crucial 
events correspond to the application of a completely positive superoperator, defined as $\tau_{D} L_{D}+I$, where $L_{D}$ is given by Eq. (24). The density matrix evolution reads [we rewrite here for the reader's convenience Eq. (6)]

$$
\frac{d}{d t} \rho(t)=L_{U} \rho(t)-\int_{0}^{t} d t^{\prime} \Phi\left(t-t^{\prime}\right) \tau_{D} L_{D} e^{\left(t-t^{\prime}\right) L_{U}} \rho\left(t^{\prime}\right),
$$

where the memory kernel is defined in the Laplace domain as $\widetilde{\Phi}(u)=u \tilde{\psi}(u) /[1-\widetilde{\psi}(u)]$. The main difference between Eqs. (58) and (10) is the unitary contribution appearing outside the time convolution. Then, even when in the natural time scale the dynamics generates a Zeno-like effect, the decay always develops oscillations, independently of the parameter values. The oscillatory behavior makes it impossible for us to interpret the relaxation as generated by renewal events. This indicates that the adoption of the new form of Eq. (10) rather than Eq. (6) is essential to establish a quantum statistical picture equivalent to the symbolic sequences generated by the BQD intermittent fluorescence.

\section{CONCLUDING REMARKS}

We have proven that Eq. (10) with the frequency of the unitary term so small as to generate in the natural time scale a symbolic sequence corresponding to the Zeno effect is statistically equivalent to the generator of the symbolic sequence discussed in Ref. 4.

Is this result relevant as a proposal to account for the main properties of blinking quantum dots? The authors of a recent article published in Physics Today ${ }^{38}$ reviewed the literature on blinking quantum dots and reached the same conclusion as the authors of another review paper, published on Nature Physics: ${ }^{17}$ There is not yet a satisfactory theory for the inverse power law distribution density of the "on light" and "off light" states. We have to stress that our conjectures on the adoption of the CTRW procedure of this article as reflecting the interaction among many units is not yet well founded. However, we did succeed in deriving a quantum mechanical master equation, Eq. (10), that is equivalent to the generator of the blinking quantum dot sequences, under the assumption that this is a renewal process.

The renewal condition seems to be well assessed by both of the research work of Brokman et al..$^{39}$ and Paradisi et al. ${ }^{26}$ Furthermore, the renewal assumption led Verberk et al. ${ }^{40}$ to a theoretical prediction of the inverse power law decay of an ensemble of blinking quantum dots agreeing with experimental results.

The work by Stefani $e t$ al. ${ }^{41}$ and the more recent work by Hoogenboon et al. ${ }^{42}$ however, reveal a significant correlation between the time duration of two consecutive light on (light off) states, thereby triggering the need, according to the authors of Ref. 38, for additional experiments and reanalysis of existing data.

How to combine memory with the inverse power law distribution of the waiting times? This is, in principle, possible by means of a combination of the model proposed by Kuno et al. ${ }^{43}$ and the superstatistics of Beck and Cohen. ${ }^{44}$ As shown in Ref. 26, the assumption that the distribution density of the Poisson transition rates is a gamma function makes this model generate an inverse power law distribution of waiting times.

On the basis of these remarks we conclude in the limiting case that the theory of superstatistics applies, the generalized quantum master equation of Eq. (10) must be replaced by

$$
\frac{d}{d t} \rho_{\tau_{D}}(t)=-\frac{l}{\hbar}\left[\hat{H}, \rho_{\tau_{D}}(t)\right]-L_{D} \rho_{\tau_{D}}(t)+\Lambda \rho_{\tau_{D}}(t) .
$$

This is along the lines of the stochastic Liouville equation proposed by Kubo many years ago. ${ }^{45}$ For recent applications of this approach, see Refs 46-48. The density matrix $\rho_{\tau_{D}}(t)$ depends on both the quantum space of interest and the space spanned by the eigenvectors of the operator $\Lambda$, which drives the fluctuating parameter $\tau_{D}$. This procedure has been successfully used for 46 years, and it is expected to work well also in this case, thereby proving that recourse can be made again of the Lindblad operator $L_{D}$.

The authors of Ref. 49 noticed that also in the case when the theory of superstatistics is assumed to work, the abrupt changes of $\tau_{D}$ may correspond to renewal events that determine the anomalous scaling of the system in the time asymptotic limit. Furthermore, if we make the assumption that the correlation time $T_{\text {corr }}$ of the correlation function $\left\langle\tau_{D}\left(t_{1}\right) \tau_{D}\left(t_{2}\right)\right\rangle$ is finite, it is possible to generate a strong correlation between two consecutive $\tau_{\text {on }}$ and two consecutive $\tau_{\text {off }}$ with no effect on the long-time renewal nature of the intermittent fluorescence process. In this case, Eq. (59) becomes equivalent to Eq. (10). Only in the case when the $T_{\text {corr }}=\infty$, it would be necessary to replace Eq. (10) with Eq. (59). A more accurate discussion of this important issue is done somewhere else. ${ }^{50}$ Here we limit ourselves to noticing that according to the theoretical analysis of Ref. 50 the longtime limit of the process of intermittent luminescence is described correctly by Eq. (10) even in the presence of the memory effects revealed by the experimental work of Refs. 41 and 42, thereby settling the puzzle of how to combine the trajectory memory with the renewal arguments that allowed the authors of Ref. 40 to predict correctly the luminescence decay of a set of BQD.

Equation (10) is based on Eq. (41), which implies that the initial condition corresponds to an event occurrence. This choice prevents us from reproducing the stationary condition even when $\mu>2$, and the stationary condition is possible (see, for instance, the work of Zharikov et al. ${ }^{51}$ ). A more general initial condition can be established by using the proposal of the recent work of Esposito and Lindenberg. ${ }^{52}$ However, we expect that this will not affect the long-time limit explored in this paper and the corresponding physical conclusions either.

Finally as a last but not least remark, we note that that the cooperation-induced subordination approach proposed in this paper may have the effect of bringing to the macroscopic level the antientropic effects discussed by the authors of Ref. 25. On the one hand, this may help the search for lasers operating without population inversion in a wider range of frequencies ${ }^{53}$ in addition to triggering the attention on the 
quantum mechanical aspects of the subordination-induced macroscopic coherence that has been recently discussed ${ }^{54}$ only within a classical context.

\section{ACKNOWLEDGMENTS}

The authors acknowledge financial support from Welch through Grant No. B-1577.

${ }^{1}$ D. C. Mikulecky, Comput. Chem. (Oxford) 25, 341 (2001).

${ }^{2}$ M. Cotsaftis, Complex Systems and Self-Organization Modelling, Understanding Complex Systems (Springer, Berlin, 2009), Chap. 1, pp. 3-19.

${ }^{3}$ S. Bianco, E. Geneston, P. Grigolini, and M. Ignaccolo, Physica A 387, 1387 (2008).

${ }^{4}$ N. Korabel and E. Barkai, Phys. Rev. Lett. 102, 050601 (2009).

${ }^{5}$ H. P. Lu, L. Xun, and X. S. Xie, Science 282, 1877 (1998).

${ }^{6}$ M. Lippitz, F. Kulzer, and M. Orrit, ChemPhysChem 6, 770 (2005).

${ }^{7}$ E. Joos, H. D. Zeh, C. Kiefer, D. Giulini, J. Kupsch, and I.-O. Stamatescu, Decoherence and the Appearance of a Classical World in Quantum Theory, 2nd ed. (Springer, Berlin, 2003).

${ }^{8}$ A. J. Leggett, S. Chakravarty, A. T. Dorsey, M. P. A. Fisher, A. Garg, and W. Zwerger, Rev. Mod. Phys. 59, 1 (1987).

${ }^{9}$ A. Suárez, R. Silbey, and I. Oppenheim, J. Chem. Phys. 97, 5101 (1992).

${ }^{10}$ V. Gorini, A. Kossakovski, and E. C. G. Sudarshan, J. Math. Phys. 17, 821 (1976).

${ }^{11}$ G. Lindblad, Commun. Math. Phys. 48, 119 (1976).

${ }^{12}$ R. J. Cook and H. J. Kimble, Phys. Rev. Lett. 54, 1023 (1985).

${ }^{13}$ W. Neuhauser, M. Hohenstatt, P. Toschek, and H. Dehmelt, Phys. Rev. Lett. 41, 233 (1978).

${ }^{14}$ W. Neuhauser, M. Hohenstatt, P. E. Toschek, and H. Dehmelt, Phys. Rev. A 22, 1137 (1980).

${ }^{15}$ H. Dehmelt, X. Zhao, and N. Yu, Proc. Natl. Acad. Sci. U.S.A. 93, 6861 (1996)

${ }^{16}$ Y. Yu, S.-L. Zhu, G. Sun, X. Wen, N. Dong, J. Chen, P. Wu, and S. Han, Phys. Rev. Lett. 101, 157001 (2008)

${ }^{17}$ P. Frantsuzov, M. Kuno, B. Jankó, and R. A. Marcus, Nat. Phys. 4, 519 (2008).

${ }^{18}$ S. M. Barnett and S. Stenholm, Phys. Rev. A 64, 033808 (2001).

${ }^{19}$ J. Wilkie, Phys. Rev. E 62, 8808 (2000).

${ }^{20}$ R. Zwanzig, J. Chem. Phys. 33, 1338 (1960).

${ }^{21}$ A. A. Budini, Phys. Rev. A 69, 042107 (2004).

${ }^{22}$ A. A. Budini, Phys. Rev. E 72, 056106 (2005); A. A. Budini and H. Schomerus, J. Phys. A 38, 9251 (2005).

${ }^{23}$ E. W. Montroll and G. H. Weiss, J. Math. Phys. 6, 167 (1965).
${ }^{24}$ B. J. West, E. L. Geneston, and P. Grigolini, Phys. Rep. 468, 1 (2008).

${ }^{25}$ N. Erez, G. Gordon, M. Nest, and G. Kurizki, Nature (London) 452, 724 (2008).

${ }^{26}$ S. Bianco, P. Grigolini, and P. Paradisi, J. Chem. Phys. 112, 3177 (2005).

${ }^{27}$ V. Braginsky and F. Ya. Khalili, Quantum Measurement (Cambridge University Press, Cambridge, 1992).

${ }^{28}$ V. Čápek and I. Barvík, Physica A 294, 388 (2001).

${ }^{29}$ E. C. G. Sudarshan and B. Misra, J. Math. Phys. 18, 756 (1977).

${ }^{30}$ P. Grigolini, M. G. Pala, and L. Palatella, Phys. Lett. A 285, 49 (2001).

${ }^{31}$ E. Barkai and R. J. Silbey, J. Phys. Chem. B 104, 3866 (2000).

${ }^{32}$ R. Metzler and J. Klafter, J. Phys. Chem. B 104, 3851 (2000).

${ }^{33}$ I. M. Sokolov, Phys. Rev. E 66, 041101 (2002).

${ }^{34}$ R. Failla, P. Grigolini, M. Ignaccolo, and A. Schwettmann, Phys. Rev E 70, 010101(R) (2004).

${ }^{35}$ H. Bateman, Higher Transcendental Functions (McGraw-Hill, New York, 1953), Vol. III.

${ }^{36}$ R. B. Paris, Proc. R. Soc. London, Ser. A 458, 3041 (2002).

${ }^{37}$ A. A. Budini, Phys. Rev. A 74, 053815 (2006); H. P. Breuer, ibid. 75, 022103 (2007).

${ }^{38}$ F. Stefani, J. Hoogenboom, and E. Barkai, Phys. Today 62(2), 34 (2009).

${ }^{39}$ X. Brokmann, J.-P. Hermier, G. Messin, P. Desbiolles, J.-P. Bouchaud, and M. Dahan, Phys. Rev. Lett. 90, 120601 (2003).

${ }^{40}$ R. Verberk, J. W. M. Chon, M. Gu, and M. Orrit, Physica E (Amsterdam) 26, 19 (2005).

${ }^{41}$ F. D. Stefani, X. Zhong, W. Knoll, M. Han, and M. Kreiter, New J. Phys. 7, 197 (2005)

${ }^{42}$ J. P. Hoogenboom, J. Hernando, M. F. García-Parajó, and N. F. van Hulst, J. Phys. Chem. 112, 3417 (2008).

${ }^{43}$ M. Kuno, D. P. Fromm, H. F. Hamann, A. Gallagher, and D. J. Nesbitt, J. Chem. Phys. 115, 1028 (2001); M. Kuno, D. P. Fromm, H. F. Hamann, A. Callagher, and D. J. Nesbitt, ibid. 112, 3117 (2000).

${ }^{44}$ C. Beck, Phys. Rev. Lett. 87, 180601 (2001).

${ }^{45}$ R. Kubo, J. Math. Phys. 4, 174 (1963).

${ }^{46}$ F. Sanda and S. Mukamel, J. Phys. Chem. B 112, 14212 (2008).

${ }^{47}$ A. I. Shushin, Phys. Rev. E 78, 051121 (2008).

${ }^{48}$ D. Segal, Phys. Rev. Lett. 101, 260601 (2008).

${ }^{49}$ P. Allegrini, F. Barbi, P. Grigolini, and P. Paradisi, Phys. Rev. E 73, 046136 (2006).

${ }^{50}$ P. Paradisi and P. Grigolini (unpublished).

${ }^{51}$ A. A. Zharikov, S. I. Temkin, and A. I. Burshtein, Chem. Phys. 103, 1 (1986).

${ }^{52}$ M. Esposito and K. Lindenberg, Phys. Rev. E 77, 051119 (2008).

${ }^{53}$ K. R. Chapin and M. O. Scully, Nature (London) 452, 705 (2008).

${ }^{54}$ G. Ascolani, M. Bologna, and P. Grigolini, Physica A 388, 2727 (2009). 